\title{
Defect-Unbinding Transition in Layered Superconductors
}

\author{
Matthew J.W. Dodgson, Vadim B. Geshkenbein, and Gianni Blatter \\ Theoretische Physik, ETH-Hönggerberg, CH-8093 Zürich, Switzerland
}

(February 17, 1999)

\begin{abstract}
We establish a new interstitial-vacancy unbinding transition of the Berezinskii-KosterlitzThouless type, transforming the three dimensional pancake vortex lattice of a decoupled layered superconductor into a defected solid. This transition is the natural finite-field extension of the vortex-anti-vortex unbinding transition establishing the zero-field superfluid stiffness. At finite Josephson coupling, the defect unbinding transition turns into a topological decoupling transition.
\end{abstract}

PACS numbers: 74.60.Ec, 74.60.Ge

The soft vortex matter in high-temperature layered superconductors exhibits a fascinating rich phase diagram with a variety of phase transitions modifying the various intra- and interplanar correlations. These include a melting transition from a line solid to a line liquid 近3, a sublimation transition taking the solid into a pancake vortex gas [4], and a decoupling transition [4] [7] destroying the superconducting coherence between the planes. In this letter we concentrate on the decoupled limit where the three-dimensional (3D) nature of the vortex system is solely due to the long range electromagnetic interaction between the pancake vortices. We establish that in the crystal phase there is a Berezinskii-Kosterlitz-Thouless (BKT) phase transition which separates the pancakevortex lattice from a defected 3D solid with a finite density of free interstitials and vacancies, see Fig. 1. With finite Josephson interactions this defect-unbinding transition also triggers the decoupling of the layers, preempting other proposed mechanisms of decoupling [6,7].

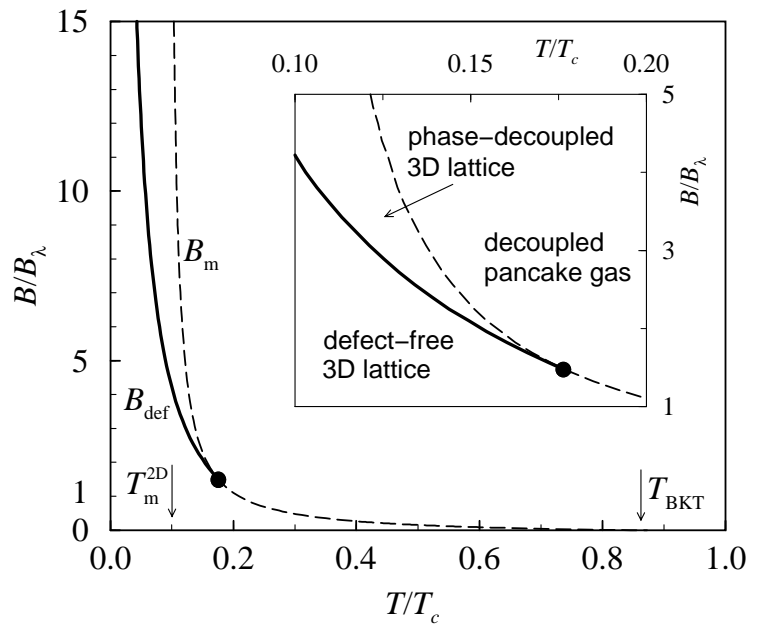

FIG. 1. Phase diagram for a weakly-coupled layered superconductor. The solid line marks the defect-unbinding transition $B_{\text {def }}(T)$ which transforms into the decoupling transition $B_{\mathrm{dec}}(T)$ in the case of a finite weak Josephson coupling with $\varepsilon<d / \lambda$. The dashed line shows the melting line $B_{\mathrm{m}}(T)$ calculated within a self-consistent analysis. (We have used parameters $\lambda(0) \approx 2000 \AA, d \approx 15 \AA$, and $T_{c} \approx 100 \mathrm{~K}$.)
The vortex lattice in layered superconductors is "soft" and melts at low temperatures; for fields $B>B_{\lambda}=$ $\Phi_{0} / \lambda^{2}(\lambda=$ penetration depth) the transition is governed by the shear interaction between pancake vortices in the same layer and thus is close to the two-dimensional dislocation-mediated BKT melting in the individual layers [8]. The 3D nature of the transition becomes explicit only at low fields $B<B_{\lambda}$, where the electromagnetic (tilt) interaction between pancake vortices in different layers dominates over the intralayer (shear) interaction due to the planar currents [4. Here, we study another thermodynamic property of the pancake-vortex lattice its susceptibility to the formation of free defects in the form of pancake-vortex interstitials and vacancies. It has recently been shown [9] for the pancake-vortex lattice in layered decoupled superconductors that the self-energy of (and the logarithmic interaction between) vacancies and interstitials is strongly screened due to the relaxation of the vortex lattice surrounding the defects. We find that the corresponding interstitial-vacancy pair creation can be understood in terms of a simple vortex-anti-vortex pair creation in a superconductor with a vortex-induced suppression of the superfluid density. The usual zero-field BKT transition establishing the superfluid stiffness of the individual superconducting layers [10] therefore has its counterpart at finite fields in the form of interstitialvacancy unbinding in the vortex solid.

We start from a rigid vortex lattice in a decoupled layered superconductor and move one pancake by the distance $R$ within the same layer to produce an interstitialvacancy defect pair. The excitation energy associated with this manipulation contains a self-, an interaction-, and a core-energy part and reads

$$
E_{i, v}(R, L)=2 \varepsilon_{0} d\left[\ln \frac{L}{a_{0}}-\ln \frac{L}{R}\right]+\left(\eta_{i}+\eta_{v}\right) \varepsilon_{0} d .
$$

Here, $\varepsilon_{0}=\left(\Phi_{0} / 4 \pi \lambda\right)^{2}$ is the basic line energy associated with the vortex, $d$ is the layer separation, $\lambda$ denotes the planar penetration depth, $a_{0}=\sqrt{\Phi_{0} / B}$ is the lattice constant, and $L$ the macroscopic extent of the layer. The nu- 
mericals $\eta_{i, v}$ quantify the core energies of the interstitialand the vacancy-defect and are of order 0.1 [11, 12].

Next, we let the lattice relax around the defect. We concentrate on a single interstitial and determine the screened self-energy - the interaction between the defects is screened in the same manner. We first ignore the electromagnetic coupling to the pancake vortices in the other layers and take them into account in a second step later. The interaction energy of the pancake-vortex configuration reads

$E_{\mathrm{int}}=\frac{1}{2} \sum_{i, j} V_{\mathrm{pc}}\left(\mathbf{R}_{i}-\mathbf{R}_{j}\right)=\frac{1}{2} \int_{\mathrm{BZ}} \frac{d^{2} K}{(2 \pi)^{2}} n_{\mathbf{K}} V_{\mathrm{pc}}(\mathbf{K}) n_{-\mathbf{K}}$

$\left(V_{\mathrm{pc}}(R)=-2 \varepsilon_{0} d \ln \left(R / a_{0}\right)\right.$ is the pancake interaction energy and $n_{\mathbf{K}}=\sum_{i} e^{i \mathbf{K} \cdot \mathbf{R}_{i}}$ the Fourier transform of the pancake-vortex density), from which one can derive the dispersive compression modulus

$$
c_{11}(K)=n^{2} V_{\mathrm{pc}}(K)=\frac{4 \pi \varepsilon_{0} d}{a_{0}^{4} K^{2}},
$$

with $n=B / \Phi_{0}$ the vortex density. The elastic energy of the lattice with one defect can be written in the form

$$
E_{\mathrm{el}}=\int_{\mathrm{BZ}} \frac{d^{2} K}{(2 \pi)^{2}}\left[\frac{c_{11}}{2}\left|\mathbf{K} \cdot \mathbf{u}_{\mathbf{K}}\right|^{2}+i n\left(\mathbf{K} \cdot \mathbf{u}_{\mathbf{K}}\right) V_{\mathrm{pc}}(K)\right],
$$

where $\mathbf{u}_{i}$ denotes the displacement field of the pancake vortex lattice, $\mathbf{u}_{\mathbf{K}}$ its Fourier transform. While the first term in (3) describes the elastic energy of the relaxed lattice, the second term originates from the force the interstitial exerts on the pancake vortices, $E_{\text {source }}=$ $\sum_{i} V\left(\mathbf{R}_{i}-\mathbf{u}_{i}\right)$ (we place the interstitial at the origin). Minimizing (3) with respect to $\mathbf{u}$ we obtain the distortion

$$
\mathbf{u}_{\mathbf{K}}=-\frac{i n \mathbf{K} V_{\mathrm{pc}}(K)}{c_{11}(K) K^{2}}=-\frac{i}{n} \frac{\mathbf{K}}{K^{2}},
$$

where we have made use of (2). The displacement $\mathbf{u}(\mathbf{R})=\mathbf{R} / 2 \pi n R^{2}$ removes exactly one pancake vortex from the neighborhood of the interstitial and thus produces perfect screening [9]. As a consequence, the logdivergent self-energy in (1) is compensated by the gain in elastic energy under the relaxation of the lattice: Inserting the solution (4) back into (3), we obtain

$$
E_{\mathrm{el}}=-\frac{1}{2} \int_{\mathrm{Bz}} \frac{d^{2} K}{(2 \pi)^{2}} V_{\mathrm{pc}}(K)=-\varepsilon_{0} d \ln \frac{L}{a_{0}} .
$$

This perfect screening is spoiled by the interaction of the pancake vortices in the layer $(n=0)$ with those in the other layers $(n \neq 0)$. Let us view the lattice as being made from columns of pancake vortices. The electromagnetic interaction of the pancake vortices in the $n=0$-layer with the rest of the lattice then can be cast into the form of a substrate potential

$$
V_{\mathrm{sub}}=\frac{1}{2} \sum_{i} \alpha_{s} u_{n=0, i}^{2}
$$

where the curvature $\alpha_{s}=\left(\varepsilon_{0} d / \lambda^{2}\right) \ln \left(a_{0} / d\right)$ follows from the electromagnetic energy associated with displacing a single vortex in a column 13.14. While the individual contribution from each pancake vortex in the column involves the prefactor $d / \lambda$ and hence is small, the longrange nature of the electromagnetic interaction leads to a large number $(\sim \lambda / d)$ of terms from distant layers. Adding the substrate energy $V_{\text {sub }}$ to the elastic energy (3) and minimizing, the displacement field (4) is reduced by the factor $1+g$ with [9]

$$
g=\frac{n \alpha_{s}}{c_{11}(K) K^{2}}=\frac{a_{0}^{2}}{4 \pi \lambda^{2}} \ln \frac{a_{0}}{d} \approx \frac{H_{c 1}}{B} .
$$

( $H_{c_{1}}$ is the lower critical field.) The screening charge is reduced to the fraction $1 /(1+g)$ of a pancake vortex and correspondingly the divergent self-energy in (11) is only partially cancelled by the lattice relaxation; inserting $\mathbf{u}_{\mathbf{K}}$ back into the expression for the elastic energy, we obtain $E_{\text {el }}=-\varepsilon_{0} d \ln \left(L / a_{0}\right) /(1+g)$. The relaxation of the pancake-vortex lattice then reduces the self-energy in (1) by the factor $g /(1+g)$. At large fields, $B>H_{c_{1}}$, where the vortex-vortex interaction is dominated by the intraplanar forces we have $g<1$ and find a large downward renormalization of the energy scale of the defects. When $g>1$ at small fields $B<H_{c_{1}}$, the interaction between the pancake vortices is dominated by the interlayer forces and the effect of screening is small.

The above analysis extends trivially to the case of a pancake-vortex vacancy as well as to the interaction between the defects. The energy for an interstitial-vacancy pancake-vortex pair of extent $R$ placed into one plane of a decoupled layered superconductor then involves the screened self- and interaction-energies

$$
E_{i, v}^{\mathrm{sc}}(R, L)=\frac{2 g \varepsilon_{0} d}{1+g} \ln \frac{R}{a_{0}}+\left(\eta_{i}^{\prime}+\eta_{v}^{\prime}\right) \varepsilon_{0} d .
$$

Before proceeding, we comment on the approximation we have made in determining the substrate potential (6), where we have ignored the relaxation of the pancakevortex positions in neighboring layers: a simple estimate shows that the relaxation $u_{n, i}$ of the pancakes in the $n$-th layer is smaller by the factor $d / \lambda, u_{n, i} \sim(d / \lambda) u_{0, i}$, and hence the correction to the substrate potential (6) is small, $\delta V_{\text {sub }, i} \sim(\lambda / d) \alpha_{s} u_{n, i}^{2} \sim(d / \lambda) \alpha_{s} u_{0, i}^{2}$.

We now discuss the implications of the result (8) for the pancake-vortex phase diagram. An immediate consequence of the logarithmic interaction between the defects is a Berezinskii-Kosterlitz-Thouless transition [10]. The field dependent screening of the interaction pushes this defect-unbinding transition to low temperatures,

$$
T_{\text {def }}(B)=\frac{g}{1+g} \frac{\varepsilon_{0} d}{2}
$$


and solving for $B$ we obtain the defect-unbinding line

$$
B_{\mathrm{def}}(T)=\frac{\Phi_{0}}{4 \pi \lambda^{2}} \ln \left(\frac{a_{0}}{d}\right)\left(\frac{\varepsilon_{0} d}{2 T}-1\right)
$$

In the presence of a finite Josephson coupling, the appearance of free defects in the solid triggers the decoupling of the superconducting layers and the defect-unbinding line $B_{\text {def }}(T)$ transforms to a decoupling line $B_{\text {dec }}(T)$. For fields above $B_{\mathrm{dec}}(T)$ the system develops a finite $c$-axis resistivity with $\rho_{c}$ proportional to the number of free mobile defects $n_{d} \propto a_{0}^{-2} \exp \left(-2 b / \sqrt{1-T / T_{\text {def }}}\right)$, see Ref. [15] ( $b$ is a non-universal constant). This topological decoupling transition corresponds to the quartet-unbinding transition first proposed by Feigel'man et al. [13. (or the supersolid transition [11]; a defect proliferation transition is also described in [16]) - here, we establish a rigorous basis for the transition via the BKT scenario, accounting for the screening response of the pancake-vortex lattice. A finite Josephson coupling does not change result (9) if the extra Josephson energy $\xi_{\text {def }}^{2} E_{\mathrm{J}}$ within the coherence area $\xi_{\text {def }}^{2} \sim n_{d}^{-1}$ remains small, hence $a_{0}^{2} \varepsilon^{2} \varepsilon_{0} / d<T_{\text {def }} \sim g \varepsilon_{0} d$, producing the condition $\varepsilon<d / \lambda$.

In the past, a number of scenarios have been proposed for the decoupling transition in layered superconductors. We have to distinguish between weak $(\lambda<\Lambda)$ and intermediate $(\Lambda<\lambda)$ coupling, where we have defined the Josephson screening length $\Lambda=d / \varepsilon$. For weak coupling we define the crossover field $B_{\times}=B_{\lambda} \Lambda^{2} / \lambda^{2}>B_{\lambda}$. Below $B_{\times}$, phase fluctuations are determined by the interlayer (tilt) interaction and the decoupling line has been predicted to take the form $B_{\mathrm{dec}}(T)=$ $B_{\lambda}\left(\varepsilon_{0} d / \pi T\right) \ln \left(a_{0} / d\right)$ [6,7]. Above $B_{\times}$, the intralayer (shear) interaction is important and the decoupling line takes the form $B_{\text {dec }}(T) \sim B_{\Lambda}\left(\varepsilon_{0} d / T\right)^{2}$ 仼. For an intermediate coupling the crossover field takes the form $B_{\times}=B_{\Lambda}=\Phi_{0} / \Lambda^{2}$ and the low and high field decoupling lines read $B_{\mathrm{dec}}(T)=B_{\Lambda}\left(\varepsilon_{0} d / e \pi T\right)<B_{\times}\|6\|$ and $B_{\text {dec }}(T) \sim B_{\Lambda}\left(\varepsilon_{0} d / T\right)^{2}>B_{\times}$.5. Parametrically, all these results are easily reproduced via a Lindemann criterion for the interlayer phase correlator [4,57. Our result for topological decoupling (10) is valid for weak coupling below $B_{\times}$and so should be compared to the results of Daemen et al. [6] and of Horovitz and Goldin [7]. Of course, whichever decoupling transition occurs first can be the only such transition, and we find that our topological decoupling line $B_{\mathrm{dec}}(T)$ is a factor 8 below the line advocated in Refs. 6,7]. This numerical relation between the two results is not fortuitous, as we will now discuss.

In fact, the result (8) and the associated transition (9) is easily understood as the finite-field extrapolation of the zero-field vortex-anti-vortex pair creation and their unbinding transition. This understanding brings forward an interesting parallel between the competing zero-field intralayer vortex unbinding- [13] and the interlayer vortex loop transition suggested by Friedel [17] on the one hand, and the present intralayer defect unbinding- and the interlayer decoupling transition advocated by Daemen et $a l$. and by Horovitz and Goldin on the other hand. In order to understand that we deal with the identical physics in both cases we start from the London free energy of a layered superconductor, $\mathcal{F}=\sum_{n} F_{n}+\int d^{3} r B^{2} / 8 \pi$ with

$F_{n}=\int d^{2} R \frac{\varepsilon_{0} d}{2 \pi}\left[\left(\nabla \varphi_{n}+\mathbf{a}\right)^{2}+\frac{2 \varepsilon^{2}}{d^{2}}\left(1-\cos \phi_{n, n+1}\right)\right]$.

Here, $\nabla=\left(\partial_{x}, \partial_{y}\right), \mathbf{a}=2 \pi \mathbf{A} / \Phi_{0}$ is the planar component of the vector potential, $\phi_{n, n+1}=\varphi_{n+1}-\varphi_{n}+\int d z a_{z}$, and $\varepsilon^{2}=m / M<1$ is the mass anisotropy ratio. For vanishing interlayer coupling, the individual layers undergo a zero-field BKT vortex unbinding transition at $T_{\mathrm{BKT}}=\varepsilon_{0} d / 2 \sqrt{13}$. On the other hand, it has been suggested by Friedel [17], that the coupled system might undergo a decoupling transition triggered by low-energy interplanar vortex loops. However, as shown by Korshunov [18], this transition would take place only at higher temperatures $T_{\mathrm{L}}=4 \varepsilon_{0} d$ and is therefore preempted by the vortex unbinding transition at $T_{\mathrm{BKT}}$.

In finite fields the presence of vortices has to be accounted for. A simple derivation of the appropriate free energy starts from the continuous elastic description of the pancake-vortex system, combining compression, shear, and tilt energies. In the incompressible limit we can drop the compression term and write the phase variable in terms of the displacement field $\mathbf{u}$, $\varphi_{\mathbf{K}}=\left(2 \pi i / a_{0}^{2}\right)\left(\mathbf{u}_{\mathbf{K}} \times \mathbf{K}\right) / K^{2}$. The electromagnetic tilt energy takes the form $g \varepsilon_{0} d(\nabla \varphi)^{2} / 2 \pi$ with $g$ given by (7). Comparing with (11), the factor $g$ accounts for the suppression of the superfluid density due to the presence of the vortices. In order to obtain the correct limit for $B \rightarrow 0$ we have to include the bare superfluid density. This is easily achieved via a calculation of the effective penetration depth $\lambda_{\text {eff }}$ in the presence of vortices, $\lambda_{\text {eff }}^{2}=\lambda^{2}+B^{2} / 4 \pi \alpha_{\mathrm{L}}$, where $\alpha_{\mathrm{L}}$ denotes the Labusch parameter describing vortex "pinning," $V_{\text {pin,i }}=\alpha_{\mathrm{L}} u_{i}^{2} / 2$. Here, "pinning" is due to the substrate potential (6) and hence $\alpha_{\mathrm{L}}=\alpha_{s} / a_{0}^{2} d=4 \pi \varepsilon_{0} g / a_{0}^{4}$. Collecting results, we obtain the suppression factor for the superfluid density $\rho_{\text {eff }} / \rho=\lambda^{2} / \lambda_{\text {eff }}^{2}=g /(1+g)$. In the end, the free energy replacing (11) in the presence of vortices takes the form

$$
\begin{aligned}
F_{n} & =\int d^{2} R \frac{\varepsilon_{0} d}{2 \pi}\left[\frac{g}{1+g}\left(\nabla \varphi_{n}+\mathbf{a}\right)^{2}+\frac{a_{0}^{4}}{16 \pi}\left(\Delta \varphi_{n}\right)^{2}\right. \\
& \left.+\frac{2 \varepsilon^{2}}{d^{2}}\left(1-\cos \phi_{n, n+1}\right)\right] .
\end{aligned}
$$

For fields $B<B_{\times}=B_{\lambda} \Lambda^{2} / \lambda^{2}$ we can drop the shear term $\propto(\Delta \varphi)^{2}$ in comparison with the electromagnetic and Josephson tilt energies. The free energy (12) then is identical in form to the one studied by Korshunov [18]; correspondingly, we can discuss two transitions: Turning the phase by $\pm 2 \pi$ we add/remove a vortex from the system and thus recover the energy ( 8 ) for the interstitialvacancy pair creation and the corresponding unbinding 
transition as given by (9). On the other hand, Friedel's loop transition takes place at a temperature $T_{L}(B)=$ $4[g /(1+g)] \varepsilon_{0} d$, which is just the result obtained by Daemen et al. [6] and by Horovitz and Goldin [7].

The pair of transitions discussed above is not unique to the vortex system discussed here - it appears in the context of the $X Y$-model (with coupling $J$ ) subject to a symmetry breaking field $\propto \cos \left[m \vartheta_{\mathbf{R}}\right]$ (here, $\vartheta_{\mathbf{R}}$ denotes the angle of the spin at the position $\mathbf{R}, m$ is an integer), where the BKT transition at $T_{\mathrm{BKT}}=\pi J / 2$ competes with the unlocking or 'roughening' transition at $T_{\mathrm{R}}=8 \pi J / m^{2}$ [19]. A similar scenario shows up in the context of the adsorption of a $2 \mathrm{D}$ crystal (elasticity $C$, lattice constant $a$ ) on a commensurate substrate (lattice constant $a / m)$, where the dislocation mediated melting transition at $T_{\mathrm{m}}=C a^{2} / 4 \pi$ competes with the depinning or 'roughening' transition at $T_{\mathrm{R}}=4 C \mathrm{a}^{2} / \pi \mathrm{m}^{2}$ [20] (in both cases the ratio between the two transition temperatures involves the factor $16 / \mathrm{m}^{2}$, twice larger than in the present layered situation). In that sense, Friedel's loop transition corresponds to the $(m=1)$ roughening transition which is preempted by a topological transition at lower temperatures.

Thermal fluctuations will soften the substrate potential and modify the result (10): At high temperatures and low fields the renormalized substrate potential (6) can be obtained from the thermal average $\alpha_{s}(T)=$ $\sum_{n}\left\langle\partial^{2} V_{n}\left(\mathbf{u}_{n}-\mathbf{u}_{0}\right) /\left(\partial u_{0, x}\right)^{2}\right\rangle_{\text {th }}$ (see Ref. [21] for details). Going over to Fourier space, the $\mathbf{K}$ component of the substrate potential is smoothed by the Debye-Waller factor $\exp \left[-K^{2}\left\langle u^{2}\right\rangle_{\mathrm{th}} / 2\right]$. The mean squared displacement is in turn determined through the substrate potential, $\left\langle u^{2}\right\rangle_{\text {th }}=2 T / \alpha_{s}$. Solving self-consistently, we find $\left\langle u^{2}\right\rangle_{\text {th }}=2 \lambda^{2} /\left[\left(\varepsilon_{0} d / 2 T\right)-1\right)$ and $\alpha_{s}(T)=\left[\varepsilon_{0} d / \lambda^{2}(0)\right](1-$ $\left.T / T_{\mathrm{BKT}}\right)$. Here, we have used a mean-field temperature dependence $\lambda^{2}(T)=\lambda^{2}(0) /\left(1-T^{2} / T_{c}^{2}\right)$, which gives a zero-field BKT transition at $T_{\mathrm{BKT}}=\varepsilon_{0}\left(T_{\mathrm{BKT}}\right) d / 2 \Longrightarrow$ $T_{\mathrm{BKT}}=\varepsilon_{0}(0) d /\left(1+\varepsilon_{0}(0) d / T_{c}\right)$. The decoupling line then has to be determined from (9) using the renormalized suppression factor $g(T)=a_{0}^{2}\left(1-T / T_{\mathrm{BKT}}\right) / 4 \pi \lambda(0)^{2}$ for the superfluid density and we obtain the final result near $T_{\mathrm{BKT}}$

$$
B_{\mathrm{def}}^{r}(T)=\frac{\Phi_{0}}{4 \pi \lambda^{2}(0)} \frac{\varepsilon_{0}(0) d}{T}\left(1-\frac{T}{T_{\mathrm{BKT}}}\right)^{2} .
$$

The position of the line $B_{\mathrm{def}}^{r}(T)$ should be compared with the melting (or sublimation) line $B_{\mathrm{m}}(T)$. Using the above self-consistent analysis we have determined the line in the $B-T$ phase diagram where the substrate potential collapses and the $3 \mathrm{D}$ vortex solid becomes unstable [21]. The numerical result is shown as the dashed line in Fig. 1, interpolating between $T_{\mathrm{BKT}}$ at zero field and the $2 \mathrm{D}$ melting temperature $T_{\mathrm{m}}^{2 \mathrm{D}} \approx \varepsilon_{0} d / 70$ at high fields. The asymptotic limit at low fields takes the form $B_{\mathrm{m}}(T)=\left(B_{\lambda} / 16 \pi\right)\left(T_{\mathrm{BKT}} / T\right)^{2}\left(1-T / T_{\mathrm{BKT}}\right)^{2}$. Comparing this with (13) we find that the melting line undercuts the defect-unbinding line at high temperatures, leaving only one (sublimation) transition at $B_{\mathrm{m}}(T)$. At low temperatures, $B_{\mathrm{def}}(T)$ is below the melting line, allowing for two transitions separating a defect-free solid, a phasedecoupled solid and a pancake gas. The defect-unbinding line $B_{\mathrm{def}}^{r}(T)$ calculated with the self-consistently softened substrate is shown as the full line in Fig. 1.

Though screening strongly reduces the interaction energy of the defects for fields $B>B_{\lambda}$, their core energy remains high. For an estimate we consult the numerical work of Frey et al. [11] and of Olive and Brandt [12], who find values of order $0.15-0.2 \varepsilon_{0}$ for the vacancy and interstitial line defects in a 3D vortex lattice, the interstitial typically being $30 \%$ cheaper in energy than the vacancy. Therefore at high fields the defect density at the unbinding transition is small, $n_{d} \propto a_{0}^{-2} \exp \left[-\lambda^{2} / a_{0}^{2}\right]$, (with more interstitials than vacancies) and the transition is weak.

In conclusion, we have established a rigorous framework for the topological decoupling transition in layered type II superconductors which is based on a defectunbinding transition of the BKT type.

We thank the Fonds National Suisse for financial support.

[1] D.R. Nelson, Phys. Rev. Lett. 60, 1973 (1988).

[2] A. Houghton, R.A. Pelcovits, and A. Sudbø, Phys. Rev. B 40, 6763 (1989).

[3] G. Blatter et al., Rev. Mod. Phys. 66, 1125 (1994).

[4] G. Blatter et al., Phys. Rev. B 54, 72 (1996).

[5] L. Glazman and A.E. Koshelev, Phys. Rev. B 43, 2835 (1991).

[6] L. Daemen et al., Phys. Rev. Lett. 70, 1167 (1993).

[7] B. Horovitz and T.R. Goldin, Phys. Rev. Lett. 80, 1734 (1998).

[8] D. Fisher, Phys. Rev. B 22, 1190 (1980).

[9] M. Slutzky, R. Mints, and E.H. Brandt, Phys. Rev. B 56, 453 (1997); E.H. Brandt, Phys. Rev. B 56, 9071 (1997).

[10] V.L. Berezinskii, Sov. Phys. JETP 32, 493 (1971); Sov. Phys. JETP 34, 610 (1972); J.M. Kosterlitz and D.J. Thouless, J. Phys. C 6, 1181 (1973).

[11] E. Frey, D.R. Nelson, and D.S. Fisher, Phys. Rev. B 49, 9723 (1994).

[12] E. Olive and E.H. Brandt, Phys. Rev. B 57, 13861 (1998).

[13] M.V. Feigel'man, V.B. Geshkenbein, and A.I. Larkin, Physica C 167, 177 (1990).

[14] At low fields, where $a_{0}>\lambda$ the lattice constant $a_{0}$ under the logarithm has to be replaced by the penetration depth $\lambda$.

[15] A. E. Koshelev, Phys. Rev. Lett. 76, 1340 (1996).

[16] H.M. Carruzzo and C.C. Yu, Phil. Mag. B 77, 1001 (1998).

[17] J. Friedel, J. Phys. France 49, 1561 (1988).

[18] S.E. Korshunov, Europhys. Lett. 11, 757 (1990).

[19] J.V. José et al., Phys. Rev. B 16, 1217 (1977).

[20] V.L. Pokrovsky and A.L. Talapov, Phys. Rev. Lett. 42, 65 (1979).

[21] M.J.W. Dodgson et al., in preparation. 